\title{
BOUNDED STATIONARY REFLECTION
}

\author{
JAMES CUMMINGS AND CHRIS LAMBIE-HANSON
}

\begin{abstract}
We prove that, assuming large cardinals, it is consistent that there are many singular cardinals $\mu$ such that every stationary subset of $\mu^{+}$reflects but there are stationary subsets of $\mu^{+}$that do not reflect at ordinals of arbitrarily high cofinality. This answers a question raised by Todd Eisworth.
\end{abstract}

\section{INTRODUCTION}

In this paper, we prove a consistency result about stationary reflection at successors of singular cardinals. Recall that, if $\kappa$ is a regular uncountable cardinal, $S$ is a stationary subset of $\kappa$, and $\beta<\kappa$ is an ordinal of uncountable cofinality, then we say that $S$ reflects at $\beta$ if $S \cap \beta$ is stationary in $\beta$. S reflects if it reflects at some ordinal $\beta<\kappa$, and $\operatorname{Refl}(\kappa)$ holds if every stationary subset of $\kappa$ reflects. Our notation is for the most part standard. [5] will serve as our primary reference for definitions and notations. If $\lambda<\kappa$ are cardinals, with $\lambda$ regular, then $S_{\lambda}^{\kappa}=\{\alpha<\kappa \mid \operatorname{cf}(\alpha)=\lambda\}$. The class of ordinals is denoted by On.

The extent of stationary reflection is a topic of considerable interest in set theory, particularly regarding the investigation of the tension existing between incompactness phenomena and canonical inner models on one hand and large cardinals and reflection principles on the other. Quoting two basic results in this vein, it is an easy consequence of $\Pi_{1}^{1}$-indescribability that, if $\kappa$ is weakly compact, then $\operatorname{Refl}(\kappa)$ holds, while Jensen [6] showed that, if $V=L$ and $\kappa$ is a regular, uncountable cardinal, then $\operatorname{Refl}(\kappa)$ holds if and only if $\kappa$ is weakly compact. Also note that, if $\kappa=\lambda^{+}$ and $\lambda$ is a regular cardinal, then $\operatorname{Refl}(\kappa)$ cannot hold, since $S_{\lambda}^{\kappa}$ is a non-reflecting stationary subset of $\kappa$.

We will be concerned with stationary reflection at successors of singular cardinals. The following fundamental result, due to Solovay [9] serves as a template for many of the proofs in this area.

Proposition 1.1. Suppose $\left\langle\kappa_{i} \mid i<\omega\right\rangle$ is an increasing sequence of supercompact cardinals, and let $\kappa_{\omega}=\sup \left(\left\{\kappa_{i} \mid i<\omega\right\}\right)$. Then $\operatorname{Refl}\left(\kappa_{\omega}^{+}\right)$holds.

Proof. Let $S \subset \kappa_{\omega}^{+}$be stationary. By shrinking $S$ if necessary, we may assume that there is $\lambda<\kappa_{\omega}$ such that $S \subseteq S_{\lambda}^{\kappa_{\omega}^{+}}$. Let $i^{*}<\omega$ be such that $\lambda<\kappa_{i^{*}}$, and let $j: V \rightarrow M$ be an elementary embedding witnessing that $\kappa_{i^{*}}$ is $\kappa_{\omega}^{+}$supercompact. In $M, j(S)$ is a stationary subset of $S_{\lambda}^{j\left(\kappa_{\omega}^{+}\right)}$. Let $\eta=\sup \left(j “ \kappa_{\omega}^{+}\right) . \eta<j\left(\kappa_{\omega}^{+}\right)$, and we claim that, in $M, j(S) \cap \eta$ is stationary in $\eta$.

Suppose this is not the case, and let $C \in M$ be a club in $\eta$ such that $C \cap j(S)=\emptyset$.

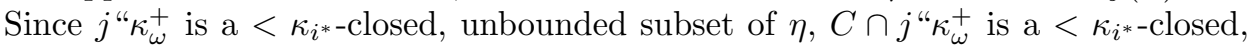

Date: January 22, 2015.

The first author was partially supported by NSF grant DMS-1101156.

The results in this paper form a part of the second author's Ph.D. thesis. 
unbounded subset of $\eta$ that is disjoint from $j(S)$. Let $D=j^{-1}$ " $\left(C \cap j^{*} \kappa_{\omega}^{+}\right)$. $D$

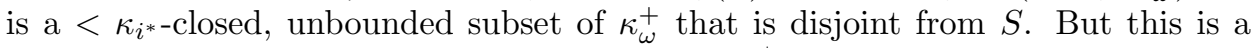
contradiction, since $S$ is a stationary subset of $S_{\lambda}^{\kappa_{\omega}^{+}}$and $\lambda<\kappa_{i^{*}}$.

Thus, $M \models " j(S)$ reflects at $\eta "$, so, by elementarity, there is $\beta<\kappa_{\omega}^{+}$such that $V \models " S$ reflects at $\beta "$.

Magidor, in [7], brings this result down to smaller cardinals by showing that, assuming the existence of $\omega$-many supercompact cardinals, it is consistent that Refl( $\left.\aleph_{\omega+1}\right)$ holds. In [8], Shelah produces, starting from a proper class of supercompact cardinals, a model in which, among other things, $\operatorname{Refl}\left(\mu^{+}\right)$holds for every singular cardinal $\mu$. In fact, he shows that, under more stringent large cardinal assumptions, such a model can contain a cardinal $\kappa$ which is $\kappa^{+n}$-supercompact for every $n<\omega$. On the other hand, he proves in the same paper that if there is a cardinal $\kappa$ that is $\kappa^{+\omega+1}$-supercompact, then there is a singular cardinal $\mu$ such that $\operatorname{Refl}\left(\mu^{+}\right)$fails. In [1], Chayut presents a simpler argument that one can force stationary reflection at the successor of every singular cardinal.

In this paper, we investigate questions about the cofinality of ordinals at which stationary sets reflect. These questions are of interest in, for example, the study of square bracket partition relations, where Eisworth has shown [4] that, if $\mu$ is a singular cardinal and $\mu^{+} \rightarrow\left[\mu^{+}\right]_{\mu^{+}}^{2}$, then, for every stationary set $S \subseteq \mu^{+}$and every regular $\lambda<\mu$, there is $\beta \in S_{>\lambda}^{\mu^{+}}$such that $S$ reflects at $\beta$. Eisworth [2] raised the natural question as to whether this is always the case assuming $\operatorname{Refl}\left(\mu^{+}\right)$holds. With this in mind, we make the following definition.

Definition Let $\mu$ be a singular cardinal. Bounded stationary reflection holds at $\mu^{+}$if $\operatorname{Refl}\left(\mu^{+}\right)$holds but there is a stationary $S \subseteq \mu^{+}$and a $\lambda<\mu$ such that $S$ does not reflect at any ordinal in $S_{\geq \lambda}^{\mu^{+}}$.

An easy argument shows that bounded stationary reflection cannot hold at $\aleph_{\omega+1}$.

Proposition 1.2. Suppose Refl $\left(\aleph_{\omega+1}\right)$ holds. Then, for every $n<\omega$, every stationary subset of $\aleph_{\omega+1}$ reflects to an ordinal $\beta$ such that $\operatorname{cf}(\beta) \geq \aleph_{n}$.

Proof. For a stationary $T \subseteq \aleph_{\omega+1}$, let $T^{\prime}=\left\{\beta<\aleph_{\omega+1} \mid T\right.$ reflects at $\left.\beta\right\}$. It is immediate that, for every stationary $T \subseteq \aleph_{\omega+1}, T^{\prime}$ is stationary. For, if not, let $C$ be club in $\aleph_{\omega+1}$ such that $C \cap T^{\prime}=\emptyset$. Then $C \cap T$ is a stationary subset of $\aleph_{\omega+1}$ that does not reflect, contradicting our hypotheses.

Now let $S \subseteq \aleph_{\omega+1}$ be stationary and let $n<\omega$. We will show that $S$ reflects at an ordinal of cofinality at least $\aleph_{n}$. Define sequences $\left\langle S_{k} \mid k<\omega\right\rangle$ and $\left\langle i_{k} \mid k<\omega\right\rangle$ as follows. Find $i_{0}<\omega$ such that $S \cap \operatorname{cof}\left(\aleph_{i_{0}}\right)$ is stationary, and let $S_{0}=S \cap \operatorname{cof}\left(\aleph_{i_{0}}\right)$. Given $S_{k}$ and $i_{k}$, find $i_{k+1}<\omega$ such that $S_{k}^{\prime} \cap \operatorname{cof}\left(\aleph_{i_{k+1}}\right)$ is stationary, and let $S_{k+1}=S_{k}^{\prime} \cap \operatorname{cof}\left(\aleph_{i_{k+1}}\right)$. Each $S_{k}$ is a stationary subset of $\aleph_{\omega+1} \cap \operatorname{cof}\left(\aleph_{i_{k}}\right)$ and, since such a set can only reflect at ordinals of cofinality greater than $\aleph_{i_{k}}$, it follows that $\left\langle i_{k} \mid k<\omega\right\rangle$ is a strictly increasing sequence.

Claim 1.3. For every $k<\omega$ and $\beta<\aleph_{\omega+1}$, if $S_{k}$ reflects at $\beta$, then $S$ reflects at $\beta$.

Proof. We proceed by induction on $k$. $k=0$ is trivial, since $S_{0} \subseteq S$. Now suppose we have proven the claim for $k$ and that $S_{k+1}$ reflects at $\beta$. Let $C$ be club in $\beta$, and let $\alpha \in C^{\prime} \cap S_{k+1}$. Then $S_{k}$ reflects at $\alpha$, so, since $C \cap \alpha$ is club in $\alpha, C \cap S_{k} \neq \emptyset$. Thus, $S_{k}$ reflects at $\beta$ and, by induction, $S$ does as well. 
Now find $i<\omega$ such that $i_{k} \geq n$. Then $S_{k}$ reflects at an ordinal $\beta$, and, by our choice of $k, \operatorname{cf}(\beta) \geq \aleph_{n}$. By the claim, $S$ also reflects at $\beta$, and we are done.

In this paper, we show that the situation is different at larger cardinals. Starting from sufficiently many supercompact cardinals, we produce a model in which bounded stationary reflection holds at many singular cardinals.

\section{Preliminaries on Approachability and Forcing}

We recall some definitions related to approachability and the ideal $I[\lambda]$. These notions were introduced by Shelah.

Definition Let $\mu$ be a singular cardinal, and let $\lambda=\mu^{+}$.

(1) Let $\vec{a}=\left\langle a_{\alpha} \mid \alpha<\lambda\right\rangle$ be a sequence of bounded subsets of $\lambda$. A limit ordinal $\beta<\lambda$ is approachable with respect to $\vec{a}$ if there is an unbounded $A \subseteq \beta$ of order type $\operatorname{cf}(\beta)$ such that, for every $\gamma<\beta$, there is $\alpha<\beta$ such that $A \cap \gamma=a_{\alpha}$.

(2) Let $S \subseteq \lambda . \quad S \in I[\lambda]$ if there is a sequence $\vec{a}=\left\langle a_{\alpha} \mid \alpha<\lambda\right\rangle$ of bounded subsets of $\lambda$ and a club $C \subseteq \lambda$ such that every $\beta \in C \cap S$ is approachable with respect to $\vec{a}$.

(3) We say that the approachability property holds at $\mu$ (written $A P_{\mu}$ ) if $\lambda \in$ $I[\lambda]$.

$I[\lambda]$ turns out to be a normal ideal on $\lambda$, and so $A P_{\mu}$ is the same as the statement that $I[\lambda]$ is an improper ideal. Also, if $\kappa<\mu$ is a regular cardinal, then $I[\lambda]$ always contains a stationary subset of $S_{\kappa}^{\lambda}$. If $\lambda^{<\lambda}=\lambda$ then, letting $\vec{a}$ and $\vec{b}$ be two enumerations of the bounded subsets of $\lambda$ in order type $\lambda$, the set $\left\{\beta \mid\left\{a_{\alpha} \mid\right.\right.$ $\left.\alpha<\beta\}=\left\{b_{\alpha} \mid \alpha<\beta\right\}\right\}$ is easily seen to be a club in $\lambda$. Thus, the set of ordinals approachable with respect to $\vec{a}$ is equal, modulo clubs, to the set of ordinals approachable with respect to $\vec{b}$. In this case, if we fix an enumeration $\vec{a}$ of the bounded subsets of $\lambda$ in order type $\lambda$, then the set $S$ of ordinals approachable with respect to $\vec{a}$ is a maximal set in $I[\lambda]$ in the sense that that if $T \subseteq \lambda$, then $T \in I[\lambda]$ if and only if $T \backslash S$ is nonstationary. If such a maximal set exists, it is referred to as the set of approachable points of $\lambda$. See [3] for proofs of these facts and other information on $I[\lambda]$.

For our forcing constructions, we will need the following definitions.

Definition Let $\mathbb{P}$ be a partial order, and let $X \subseteq \mathbb{P}$. A lower bound for $X$ is a condition $q \in \mathbb{P}$ such that, for all $p \in X, q \leq p$. If there is a unique condition $r \in \mathbb{P}$ such that $r$ is a lower bound for $X$ and, if $q$ is a lower bound for $X$, then $q \leq r$, then this condition $r$ is denoted by $\inf (X)$. If there is no such condition, then $\inf (X)$ is undefined.

Definition Let $\mathbb{P}$ be a partial order and let $\beta$ be an ordinal.

(1) The two-player game $G_{\beta}(\mathbb{P})$ is defined as follows: Players I and II alternately play entries in $\left\langle p_{\alpha} \mid \alpha<\beta\right\rangle$, a decreasing sequence of conditions in $\mathbb{P}$ with $p_{0}=1_{\mathbb{P}}$. Player I plays at odd stages, and Player II plays at even stages (including all limit stages). If there is an even stage $\alpha<\beta$ at which Player II cannot play, then Player I wins. Otherwise, Player II wins.

(2) $G_{\beta}^{*}(\mathbb{P})$ is defined just as $G_{\beta}(\mathbb{P})$ except Player II no longer plays at limit stages. Instead, if $\alpha<\beta$ is a limit ordinal, then $p_{\alpha}=\inf \left(\left\{p_{\gamma} \mid \gamma<\alpha\right\}\right)$ if 
such a condition exists. If, for some limit $\alpha<\beta$, such a condition does not exist, then Player I wins. Otherwise, Player II wins.

(3) $\mathbb{P}$ is said to be $\beta$-strategically closed if Player II has a winning strategy for the game $G_{\beta}(\mathbb{P}) . \mathbb{P}$ is said to be strongly $\beta$-strategically closed if Player II has a winning strategy for the game $G_{\beta}^{*}(\mathbb{P})$. The notions of $<\beta$-strategically closed and strongly $<\beta$-strategically closed are defined in the obvious way.

The following facts will be crucial for us.

Fact 2.1. Let $\mathbb{P}$ be a partial order and let $\kappa$ be a cardinal. If $\mathbb{P}$ is $(\kappa+1)$-strategically closed, then forcing with $\mathbb{P}$ does not add any new $\kappa$-sequences of ordinals.

Fact 2.2. [7] Let $\kappa$ be a regular cardinal, and let $\kappa<\lambda<\mu$. Suppose that, in $V^{\mathrm{Coll}(\kappa,<\lambda)}, \mathbb{P}$ is a separative, strongly $\kappa$-strategically closed partial order and $|\mathbb{P}|<\mu$. Let $i$ be the natural complete embedding of $\operatorname{Coll}(\kappa,<\lambda)$ into $\operatorname{Coll}(\kappa,<\mu)$ (namely, the identity embedding). Then $i$ can be extended to a complete embedding $j$ of $\operatorname{Coll}(\kappa,<\lambda) * \mathbb{P}$ into $\operatorname{Coll}(\kappa,<\mu)$ so that the quotient forcing $\operatorname{Coll}(\kappa,<$ $\mu) / j[\operatorname{Coll}(\kappa,<\lambda) * \mathbb{P}]$ is $\kappa$-closed.

Fact 2.3. Let $\kappa$ be a regular cardinal and let $\left\langle\mathbb{P}_{i}, \dot{\mathbb{Q}}_{j} \mid j<\alpha, i \leq \alpha\right\rangle$ be a forcing iteration in which inverse limits are taken at all limit stages of cofinality $<\kappa$ and such that, for all $i<\alpha, \Vdash_{\mathbb{P}_{i}}$ " $\dot{\mathbb{Q}}_{i}$ is (strongly) $\kappa$-strategically closed". Then $\mathbb{P}_{\alpha}$ is (strongly) $\kappa$-strategically closed.

We now define a forcing notion to shoot a club through the set of approachable points of the successor of a singular cardinal. This forcing poset is a key component of Chayut's proof of the consistency of stationary reflection in [1], and we will use it in a similar way here. Suppose $\mu$ is singular, $\lambda=\mu^{+}$, and $\lambda^{<\lambda}=\lambda$. Let $\vec{a}$ be an enumeration of the bounded subsets of $\lambda$ in order type $\lambda$, and let $S$ be the set of ordinals that are approachable with respect to $\vec{a}$. Define $\mathbb{Q}_{\vec{a}}$ to the be the poset consisting of closed, bounded subsets of $S$, where, if $p, q \in \mathbb{Q}_{\vec{a}}$, then $p \leq q$ if and only if $p$ is an end-extension of $q$.

Lemma 2.4. $\mathbb{Q}_{\vec{a}}$ is strongly $<\lambda$-strategically closed.

Proof. Let $\beta<\lambda$. We describe a winning strategy for Player II in $G_{\beta}^{*}\left(\mathbb{Q}_{\vec{a}}\right)$. Fix a large, regular cardinal $\theta$. In the course of the game, as the plays $\left\langle q_{\alpha} \mid \alpha<\beta\right\rangle$ are made, we will be defining a continuous, internally-approachable chain $\left\langle M_{\alpha} \mid \alpha<\beta\right\rangle$ of elementary submodels of $H(\theta)$ subject to the following conditions:

- $\mathbb{Q}_{\vec{a}} \in M_{0}, \beta \subset M_{0}$, and, for every limit $\alpha<\beta$, there is a club $C_{\alpha} \subseteq \alpha$ of order type $\operatorname{cf}(\alpha)$ such that $C_{\alpha} \in M_{0}$.

- For all $\alpha<\beta,\left|M_{\alpha}\right|<\lambda$ and $M_{\alpha} \cap \lambda \in \lambda$.

- For all $\alpha<\beta,\left\langle q_{\gamma} \mid \gamma \leq \alpha\right\rangle \in M_{\alpha+1}$.

If $\alpha$ is an even successor ordinal and $\left\langle q_{\gamma} \mid \gamma<\alpha\right\rangle$ has been played, then Player II plays a $q_{\alpha}$ such that

- $q_{\alpha} \in M_{\alpha}$.

- $q_{\alpha} \leq q_{\alpha-1}$.

- $\max \left(q_{\alpha}\right)>M_{\alpha-1} \cap \lambda$.

This is possible, since $S$ is stationary in $\lambda$ and $M_{\alpha}$ contains all relevant information needed to find such a $q_{\alpha}$.

Now suppose that $\alpha<\beta$ is a limit ordinal and that $\left\langle q_{\gamma} \mid \gamma<\alpha\right\rangle$ has been played. We show that $\bigcup_{\gamma<\alpha} q_{\gamma} \cup\left\{\left(M_{\alpha} \cap \lambda\right)\right\}$ is a valid condition, thus completing 
the proof. It suffices to show that $M_{\alpha} \cap \lambda$ is approachable with respect to $\vec{a}$. To this end, consider $D=\left\{M_{\gamma} \cap \lambda \mid \gamma \in C_{\alpha}\right\}$. D is a club in $M_{\alpha} \cap \lambda$ of order type $\operatorname{cf}(\alpha)=\operatorname{cf}\left(M_{\alpha} \cap \lambda\right)$. Also, every initial segment of $D$ is in $M_{\alpha}$, since every initial segment can be calculated from $C_{\alpha}$ and a sufficient initial segment of $\left\langle M_{\gamma} \mid \gamma<\alpha\right\rangle$, which are in $M_{\alpha}$. But $M_{\alpha}=$ " $\vec{a}$ is an enumeration of the bounded subsets of $\lambda$ ", so, for every $\eta<M_{\alpha} \cap \lambda$, there is $\xi<M_{\alpha} \cap \lambda$ such that $D \cap \eta=a_{\xi}$. Thus, $D$ witnesses that $M_{\alpha} \cap \lambda$ is approachable with respect to $\vec{a}$.

We now introduce a forcing notion to add a particular type of stationary set to the successor of a singular cardinal. Let $\mu>\aleph_{\omega}$ be a singular cardinal, let $\lambda=\mu^{+}$, and let $\kappa<\mu$ be a regular uncountable cardinal. $\mathbb{S}_{\lambda, \kappa}$ is a forcing poset whose conditions are of the form $p=\left(s^{p}, \gamma^{p}\right)$, where $s^{p}$ is a bounded subset of $S_{\omega}^{\lambda}$ such that, for all $\beta \in S_{\geq_{\kappa}}^{\lambda}, s^{p} \cap \beta$ is not stationary in $\beta$ and $\gamma^{p}<\lambda$ is such that $s^{p} \subseteq \gamma^{p}$. If $p, q \in \mathbb{S}_{\lambda, \kappa}$, then $p \leq q$ if and only if $\gamma^{p} \geq \gamma^{q}$ and $s^{p} \cap \gamma^{q}=s^{q}$. It is immediate that forcing with $\mathbb{S}_{\lambda, \kappa}$ adds a stationary subset of $S_{\omega}^{\lambda}$ that does not reflect at any $\beta \in S_{\geq \kappa}^{\lambda}$ and that $\mathbb{S}_{\lambda, \kappa}$ is $\kappa$-closed. We will be interested mostly in forcings of the form $\mathbb{S}_{\lambda, \aleph_{\omega+1}}$, which we will denote simply as $\mathbb{S}_{\lambda}$.

Lemma 2.5. $\mathbb{S}_{\lambda, \kappa}$ is $<\lambda$-strategically closed.

Proof. Let $\beta<\lambda$. We describe a winning strategy for Player II in $G_{\beta}\left(\mathbb{S}_{\lambda, \kappa}\right)$. In the course of the game, as the conditions $\left\langle p_{\alpha} \mid \alpha<\beta\right\rangle$ are being played, we define closed, bounded subsets $\left\langle C_{\alpha}\right| \alpha<\beta$ is even $\rangle$ of $\lambda$, ensuring that

- For all even $\alpha<\alpha^{\prime}<\beta, C_{\alpha^{\prime}}$ end-extends $C_{\alpha}$.

- For all even $\alpha<\beta$ and all $\gamma \leq \alpha, C_{\alpha} \cap s^{p_{\gamma}}=\emptyset$.

- For all even $\alpha<\beta, \gamma^{p_{\alpha}}=\max \left(C_{\alpha}\right)+1$.

Suppose that $\alpha<\beta$ is an even successor ordinal and $\left\langle p_{\gamma} \mid \gamma<\alpha\right\rangle$ has been played. Player II finds $\eta_{\alpha}>\gamma^{p_{\alpha-1}}$, lets $C_{\alpha}=C_{\alpha-2} \cup\left\{\eta_{\alpha}\right\}$, and plays $p_{\alpha}=\left(s^{p_{\alpha-1}}, \eta_{\alpha}+1\right)$. If $\alpha<\beta$ is a limit ordinal, let $\eta_{\alpha}=\sup \left(\left\{\max \left(C_{\gamma}\right) \mid \gamma<\alpha\right\}\right)$. Player II lets $C_{\alpha}=\bigcup_{\gamma<\alpha} C_{\gamma} \cup\left\{\eta_{\alpha}\right\}$ and plays $p_{\alpha}=\left(\bigcup_{\gamma<\alpha} s^{p_{\gamma}}, \eta_{\alpha}+1\right) . \quad p_{\alpha} \in \mathbb{S}_{\lambda, \kappa}$, since $\bigcup_{\gamma<\alpha} C_{\gamma}$ witnesses that $s_{\alpha} \cap \eta_{\alpha}$ is not stationary in $\eta_{\alpha}$.

Remark Note that the proof of Lemma 2.5 does not yield the stronger conclusion that $\mathbb{S}_{\lambda, \kappa}$ is strongly $<\lambda$-strategically closed. The reason is that, at limit stages $\alpha$, it was necessary to take $\gamma^{p_{\alpha}}$ to be $\eta_{\alpha}+1$ instead of $\eta_{\alpha}$, which would have been the value were we obliged to take a greatest lower bound of the conditions played thus far. It is important for the proof that $\eta_{\alpha}$ be excluded from the generic stationary set. The fact that $\mathbb{S}_{\lambda, \kappa}$ is not strongly $<\lambda$-strategically closed is one of the major obstacles that will need to be overcome in the proof of the main theorem. We do, however, get a certain amount of strong strategic closure.

Lemma 2.6. $\mathbb{S}_{\lambda, \kappa}$ is strongly $<\kappa$-strategically closed.

Proof. Since $\mathbb{S}_{\lambda, \kappa}$ is $\kappa$-closed, it suffices to show that, if $\beta<\kappa$ and $\left\langle p_{\alpha} \mid \alpha<\beta\right\rangle$ is a decreasing sequence of conditions from $\mathbb{S}_{\lambda, \kappa}$, then $\left\langle p_{\alpha} \mid \alpha<\beta\right\rangle$ has a greatest lower bound. Define $q=\left(s^{q}, \gamma^{q}\right)$ by letting $s^{q}=\bigcup_{\alpha<\beta} s^{p_{\alpha}}$ and $\gamma^{q}=\sup \left(\left\{\gamma^{p_{\alpha}} \mid \alpha<\beta\right\}\right)$. Then $q$ is easily seen to be the greatest lower bound of $\left\langle p_{\alpha} \mid \alpha<\beta\right\rangle$.

We recall one more important fact, due to Shelah, before we state our theorem.

Fact 2.7. Let $\mu$ and $\kappa$ be cardinals. Suppose that $A P_{\kappa}$ holds, $S$ is a stationary subset of $S_{<\mu}^{\kappa^{+}}$, and $\mathbb{P}$ is a $\mu$-closed forcing poset. Then $S$ remains stationary in $V^{\mathbb{P}}$. 


\section{The Main Theorem}

Theorem 3.1. Suppose there is a proper class of supercompact cardinals and GCH holds. Then there is a forcing extension in which, for every singular cardinal $\mu>\aleph_{\omega}$ that is not a cardinal fixed point, every stationary subset of $\mu^{+}$reflects but there is a stationary $S \subseteq S_{\omega}^{\mu^{+}}$such that $S$ does not reflect at any ordinal $\beta \in S_{\geq \aleph_{\omega+1}}^{\mu^{+}}$.

Proof. Let $\left\langle\kappa_{i} \mid i \in \mathrm{On}\right\rangle$ be an increasing, continuous sequence of cardinals such that

- $\kappa_{0}=\omega$.

- If $\kappa_{i}$ is singular, then $\kappa_{i+1}=\kappa_{i}^{+}$.

- If $\kappa_{i}$ is regular, then $\kappa_{i+1}$ is supercompact.

We may assume without loss of generality that, if $i$ is a limit ordinal, then $\kappa_{i}$ is singular, for, if this is not the case, then we may work not in $V$ but in $V_{\kappa_{i^{*}}}$, where $i^{*}$ is the least limit ordinal $i$ for which $\kappa_{i}$ is regular.

We now define a class forcing $\left\langle\mathbb{P}_{i}\right| i \in$ On $\rangle$ such that:

- If $i$ is a limit ordinal, then $\mathbb{P}_{i}$ is the inverse (i.e. full support) limit of $\left\langle\mathbb{P}_{j} \mid j<i\right\rangle$.

- If $i$ is a successor ordinal, then $\mathbb{P}_{i+1}=\mathbb{P}_{i} * \operatorname{Coll}\left(\kappa_{i},<\kappa_{i+1}\right)$.

- If $i>\omega$ is a limit ordinal and $i<\kappa_{i}$, then let $\dot{\vec{a}}$ be a $\mathbb{P}_{i}$-name for an enumeration of the bounded subsets of $\kappa_{i+1}$ in order type $\kappa_{i+1}$ and let $\mathbb{P}_{i+1}=\mathbb{P}_{i} * \dot{\mathbb{Q}}_{\vec{a}} * \dot{\mathbb{S}}_{\kappa_{i+1}}$. For technical reasons, we assume that, for every $j<i$, there is a set $A_{j}^{i} \subseteq \kappa_{i+1}$ in the ground model such that it is forced to be the case that all bounded subsets of $\kappa_{i+1}$ in $V^{\mathbb{P}_{j}}$ are enumerated in $\dot{\vec{a}}$ by indices in the set $A_{j}^{i}$ in a way that is defined in $V^{\mathbb{P}_{j}}$.

- If $i$ is a limit ordinal and $i=\kappa_{i}$, then $\mathbb{P}_{i+1}=\mathbb{P}_{i} *\{\mathbb{1}\}$, where $\{\mathbb{1}\}$ is trivial forcing.

For ordinals $i<k$, let $\dot{\mathbb{P}}_{i, k}$ be such that $\mathbb{P}_{k}=\mathbb{P}_{i} * \dot{\mathbb{P}}_{i, k}$. We think of conditions in $\mathbb{P}_{i}$ as being functions $p$ with domain $i$. If $\ell<i$ is a limit ordinal, $\mathbb{P}_{\ell, \ell+1}$ is of the form $\dot{\mathbb{Q}}_{\vec{a}} * \dot{\mathbb{S}}_{\kappa_{\ell+1}}$, and $p \in \mathbb{P}_{i}$, then $p(\ell)$ is thought of as a pair $\left(p(\ell)_{0}, p(\ell)_{1}\right)$ in the natural way. Note that, by Lemmas 2.4 and 2.5 and Fact 2.3 , for all ordinals $i<k$, in $V^{\mathbb{P}_{i}}$ we have that $\dot{\mathbb{P}}_{i, k}$ is $<\kappa_{i}$-strategically closed and hence does not add any new sequences of ordinals of length less than $\kappa_{i}$. Thus, for $i<k,\left(H\left(\kappa_{i}\right)\right)^{V^{\mathbb{P}_{k}}}=\left(H\left(\kappa_{i}\right)\right)^{V^{\mathbb{P}_{i}}}$, so $V^{\mathbb{P}}=\bigcup_{i \in \text { On }} V^{\mathbb{P}_{i}}$ is a model of ZFC.

We now show, by induction on ordinals $i$, that all of the $\kappa_{i}$ 's remain cardinals in $V^{\mathbb{P}}$. It is clear that there can be no other cardinals in $V^{\mathbb{P}}$ and so, when we have shown this, it will follow that $\kappa_{i}=\left(\aleph_{i}\right)^{V^{\mathbb{P}}} . \kappa_{0}=\omega$, so there is nothing to worry about here. We first consider cardinals $\kappa_{i+1}$, where $i$ is a successor ordinal or 0 . In this case, since $\left|\mathbb{P}_{i}\right|<\kappa_{i+1}, \kappa_{i+1}$ remains supercompact in $V^{\mathbb{P}_{i}}$. Since $\mathbb{P}_{i+1}=\mathbb{P}_{i} * \operatorname{Coll}\left(\kappa_{i},<\kappa_{i+1}\right), \kappa_{i+1}=\left(\kappa_{i}^{+}\right)^{\mathbb{P}_{i+1}}$. Finally, since for all $k>i+1, \mathbb{P}_{i+1, k}$ is $<\kappa_{i+1}$-strategically closed, $\kappa_{i+1}$ remains a cardinal in $V^{\mathbb{P}_{k}}$ for all $k$ and hence in $V^{\mathbb{P}}$.

If $i$ is a limit ordinal, then, by the previous paragraph, $\kappa_{i}$ is, in $V^{\mathbb{P}}$, the limit of cardinals and hence a cardinal. Finally, consider $\kappa_{i+1}$, where $i$ is a limit ordinal. Since, for all $k>i+1, \mathbb{P}_{i+1, k}$ is $<\kappa_{i+1}$-strategically closed, it suffices to show that $\kappa_{i+1}$ remains a cardinal in $V^{\mathbb{P}_{i+1}}$. Suppose this is not the case. Then there is $i_{0}<i$ such that $\left(\operatorname{cf}\left(\kappa_{i+1}\right)\right)^{V^{\mathbb{P}_{i+1}}}=\kappa_{i_{0}}$. Since $\mathbb{P}_{i_{0}+1, i+1}$ is $\kappa_{i_{0}}+1$-strategically closed, it 
must be the case that $\left(\operatorname{cf}\left(\kappa_{i+1}\right)\right)^{V^{\mathbb{P}} i_{0}+1}=\kappa_{i_{0}}$. But $\left|\mathbb{P}_{i_{0}+1}\right|<\kappa_{i}$, so $\kappa_{i+1}$ remains a regular cardinal in $V^{\mathbb{P}_{i_{0}+1}}$. This is a contradiction, so in fact $\kappa_{i+1}$ remains a cardinal in $V^{\mathbb{P}_{i+1}}$.

It remains to show that, in $V^{\mathbb{P}}$, for every limit ordinal $i>\omega$ that is not a cardinal fixed point, every stationary subset of $\kappa_{i+1}$ reflects but there is a stationary $S_{i} \subseteq S_{\omega}^{\kappa_{i+1}}$ that does not reflect at any ordinal in $S_{\geq \aleph_{\omega+1}}^{\kappa_{i+1}}$. We prove the latter statement first, since it is almost immediate. Let $i>\omega$ be such an ordinal, and let $S_{i}$ be the stationary set added by $\mathbb{S}_{\kappa_{i+1}}$. By the definition of $\mathbb{S}_{\kappa_{i+1}}$, in $V^{\mathbb{P}_{i+1}}, S_{i}$ is a stationary subset of $S_{\omega}^{\kappa_{i+1}}$ that does not reflect at any ordinal in $S_{\geq \aleph_{\omega+1}}^{\kappa_{i+1}}$. The fact that $S_{i}$ does not reflect at any such ordinal is clearly preserved in any further forcing extension. Also, since $A P_{\kappa_{i}}$ holds in $V^{\mathbb{P}_{i+1}}$ and, for all $j>i+1, \mathbb{P}_{i+1, j}$ is countably closed, $S_{i}$ remains stationary in every $V^{\mathbb{P}_{j}}$ and hence in $V^{\mathbb{P}}$. Thus, $S_{i}$ is as desired.

The following lemma, which will be useful in proving that all stationary subsets of $\kappa_{i+1}$ reflect, comes from [1]. We thank Menachem Magidor and Yair Chayut for communicating it to us.

Lemma 3.2. Let $i \in \mathrm{On}$, and let $X_{i}$ be the set of limit ordinals $k<i$ such that $\omega<k<\kappa_{k}$. Then the set $D_{i}$ of $p \in \mathbb{P}_{i}$ such that there is a function $g \in \prod_{k \in X_{i}} \kappa_{k+1}$ such that $g \in V$ and, for all $k \in X_{i}, p\left\lceil k \Vdash_{\mathbb{P}_{k}} " \max \left(p(k)_{0}\right)<g(k) "\right.$ and $p \uparrow$ $k \frown p(k)_{0} \Vdash_{\mathbb{P}_{k} * \mathbb{Q}_{\vec{a}}}$ " $\gamma^{p(k)_{1}}<g(k)$ " is dense in $\mathbb{P}_{i}$.

Proof. We actually prove the following stronger statement: Let $i_{0}<i$ be ordinals, let $X_{i_{0}, i}$ be the set of limit ordinals $k \in\left[i_{0}, i\right)$ such that $\omega<k<\kappa_{k}$, and work in $V^{\mathbb{P}_{i_{0}}}$. Let $D_{i_{0}, i}$ be the set of $p \in \mathbb{P}_{i_{0}, i}$ such that there is a function $g \in \prod_{k \in X_{i_{0}, i}} \kappa_{k+1}$ such that $g \in V^{\mathbb{P}_{i_{0}}}$ and, for all $k \in X_{i_{0}, i}, p \uparrow\left[i_{0}, k\right) \Vdash_{\mathbb{P}_{i_{0}, k}} " \max \left(p(k)_{0}\right)<g(k) "$ and $p \uparrow\left[i_{0}, k\right) \frown p(k)_{0} \Vdash_{\mathbb{P}_{i_{0}, k} * \mathbb{Q}_{\vec{a}}}$ " $\gamma^{p(k)_{1}}<g(k)$ ". Then $D_{i_{0}, i}$ is dense in $\mathbb{P}_{i_{0}, i}$.

We proceed by induction on $i$. Thus, fix ordinals $i_{0}<i$. First, suppose $i=$ $k+1$. If $k$ is a successor ordinal or $k$ is limit and $k=\kappa_{k}$, then the conclusion is trivial, since $k \notin X_{i_{0}, i}$ in this case. Thus, assume $k$ is a limit ordinal and $k<\kappa_{k}$. Work in $V^{\mathbb{P}_{i_{0}}}$ and let $p \in \mathbb{P}_{i_{0}, i}$. Find $p^{\prime} \leq p \uparrow\left[i_{0}, k\right) \frown p(k)_{0}$ and $\eta<\kappa_{i+1}$ such

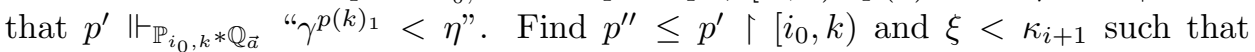
$p^{\prime \prime} \Vdash_{\mathbb{P}_{i_{0}, k}}$ " $\max \left(p^{\prime}(k)_{0}\right)<\xi "$. Finally, by the inductive hypothesis, find $p^{*} \leq p^{\prime \prime}$ such that $p^{*} \in D_{i_{0}, k}$ as witnessed by $h \in \prod_{\ell \in X_{i_{0}, k}} \kappa_{\ell+1}$. Form $\bar{p} \in \mathbb{P}_{i_{0}, i}$ by letting $\bar{p}=p^{*} p^{\prime}(k)_{0} \frown p(k)_{1}$, and define $g \in \prod_{\ell \in X_{i}} \kappa_{\ell+1}$ by letting $g(\ell)=h(\ell)$ for $\ell<k$ and $g(k)=\max (\{\eta, \xi\})$. Now $\bar{p} \leq p$, and $\bar{p} \in D_{i_{0}, i}$, as witnessed by $g$.

Now suppose $i$ is a limit ordinal, work in $V^{\mathbb{P}_{i_{0}}}$, and let $p \in \mathbb{P}_{i_{0}, i}$. Recall that $\kappa_{i}$ is singular and that $\operatorname{cf}\left(\kappa_{i}\right)=\operatorname{cf}(i)$. Let $\left\langle\ell_{\alpha} \mid \alpha<\operatorname{cf}(i)\right\rangle$ be increasing and cofinal in $i$, with $\ell_{0} \geq i_{0}$ a successor ordinal and $\kappa_{\ell_{0}}>\operatorname{cf}(i)$. Move to $V^{\mathbb{P} \ell_{0}}$, assuming that $p \uparrow\left[i_{0}, \ell_{0}\right)$ is in the generic subset of $\mathbb{P}_{i_{0}, \ell_{0}}$. Note that $\mathbb{P}_{\ell_{0}, i}$ is $\operatorname{cf}(i)+1$-strategically closed. We will play the first $\mathrm{cf}(i)$-many moves of the game $G_{\mathrm{cf}(i)+1}\left(\mathbb{P}_{\ell_{0}, i}\right)$ to produce $\left\langle p_{\alpha} \mid \alpha<\operatorname{cf}(i)\right\rangle$. Player II will play her winning strategy, and Player I will play $p \uparrow\left[\ell_{0}, i\right)$ on her first move and, on her $\alpha^{\text {th }}$ move, will play $q$ such 
that $q \uparrow\left[\ell_{0}, \ell_{\alpha}\right) \in D_{\ell_{0}, \ell_{\alpha}}$ as witnessed by $h_{\alpha} \in \prod_{k \in X_{\ell_{0}, \ell_{\alpha}}} \kappa_{k+1} .\left\langle p_{\alpha} \mid \alpha<\operatorname{cf}(i)\right\rangle$ has a lower bound $p^{*}$, and, moreover, we may assume that, for each $k \in X_{\ell_{0}, i}$, $p^{*}\left\lceil\left[\ell_{0}, k\right) \Vdash_{\mathbb{P}_{\ell_{0}, k}} " p^{*}(k)_{0}\right.$ is the greatest lower bound of $\left\langle p_{\alpha}(k)_{0} \mid \alpha<\operatorname{cf}(i)\right\rangle "$ and $p^{*}\left\lceil\left[\ell_{0}, k\right) \frown p^{*}(k)_{0} \Vdash_{\mathbb{P}_{\ell_{0}, k} * \mathbb{Q}_{\vec{a}}}\right.$ " $p^{*}(k)_{1}$ is the greatest lower bound of $\left\langle p_{\alpha}(k)_{1}\right| \alpha<$ $\operatorname{cf}(i)\rangle "$. Thus, defining $h^{*} \in \prod_{k \in X_{\ell_{0}, i}} \kappa_{k+1}$ by $h^{*}(k)=\sup \left(\left\{h_{\alpha}(k) \mid \alpha<\operatorname{cf}(i)\right\}\right)$, we can assure that $p^{*} \in D_{\ell_{0}, i}$ as witnessed by $h^{*}$.

Now move back to $V^{\mathbb{P}_{i_{0}}}$, let $\dot{p}^{*}$ be a $\mathbb{P}_{i_{0}, \ell_{0}}$-name for $p^{*}$, and let $\dot{h}^{*}$ be a $\mathbb{P}_{i_{0}, \ell_{0}}$-name for $h^{*}$. Since $\mathbb{P}_{i_{0}, \ell_{0}}$ satisfies the $\kappa_{\ell_{0}}^{+}$-c.c., we can find $h \in V^{\mathbb{P}_{i_{0}}}$ such that $\Vdash_{\mathbb{P}_{i_{0}, \ell_{0}}} " \dot{h}^{*}<$ $h "$. Thus, there is $q \leq p\left\lceil\left[i_{0}, \ell_{0}\right)\right.$ such that $q \Vdash_{\mathbb{P}_{i_{0}, \ell_{0}}}$ " $\dot{p}^{*} \in D_{\ell_{0}, i}$ as witnessed by $h "$. Find $q^{\prime} \leq q$ and $h^{\prime} \in \prod_{k \in X_{i_{0}, \ell_{0}}} \kappa_{k+1}$ such that $q^{\prime} \in D_{\ell_{0}}$ as witnessed by $h^{\prime}$. Finally, let $\bar{p} \in \mathbb{P}_{i_{0}, i}$ be such that $\bar{p}\left\lceil\left[i_{0}, \ell_{0}\right)=q^{\prime}\right.$ and $q^{\prime} \Vdash_{\mathbb{P}_{i_{0}, \ell_{0}}}$ " $\bar{p}\left\lceil\left[\ell_{0}, i\right)=\dot{p}^{*}\right.$, and let $g=h^{\prime \frown h}$. Then $\bar{p} \leq p$ and $\bar{p} \in D_{i_{0}, i}$, as witnessed by $g$.

Fix a limit ordinal $i>\omega$ such that $i$ is not a cardinal fixed point in $V^{\mathbb{P}}$. We will show that every stationary subset of $\kappa_{i+1}$ reflects. Since every subset of $\kappa_{i+1}$ in $V^{\mathbb{P}}$ appears in $V^{\mathbb{P}_{i+2}}$, it suffices to prove stationary reflection in $V^{\mathbb{P}_{i+2}}$. Let $G$ be $\mathbb{P}_{i+2}$-generic over $V$. For $i_{0}<i+2$, let $G_{i_{0}}$ be the $\mathbb{P}_{i_{0}}$-generic filter induced by $G$. Let $T \subseteq \kappa_{i+1}$ be stationary in $V[G]$. Without loss of generality, there is a successor ordinal $k<i$ such that $T \subseteq S_{\kappa_{k}}^{\kappa_{i+1}}$. We will show that $T$ reflects. The proof breaks into two cases.

Case 1: $k<\omega$.

Let $k^{*}<\omega$ be such that $k<k^{*}$, and let $i^{*}=k^{*}+1$. In $V\left[G_{k^{*}}\right], \kappa_{i^{*}}$ is supercompact. Let $j: V\left[G_{k^{*}}\right] \rightarrow M\left[G_{k^{*}}\right]$ witness that $\kappa_{i^{*}}$ is $\kappa_{i+2^{-}}$-supercompact. $j\left(\mathbb{P}_{k^{*}, i^{*}}\right)=\operatorname{Coll}\left(\kappa_{k^{*}},<j\left(\kappa_{i^{*}}\right)\right)$ and $\mathbb{P}_{i^{*}, i+2}$ is strongly $\kappa_{k^{*}}$-strategically closed, so,

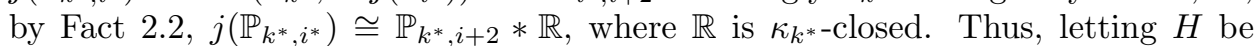
$\mathbb{R}$-generic over $V[G]$, we can extend $j$ to $j: V\left[G_{i^{*}}\right] \rightarrow M[G * H]$

We would like to extend $j$ further to have domain $V[G]$. Let $G^{i^{*}}$ be the $\mathbb{P}_{i^{*}, i+2^{-}}$ generic filter over $V\left[G_{i^{*}}\right]$ induced by $G$. We recursively build a condition $p^{*} \in$ $j\left(\mathbb{P}_{i^{*}, i+2}\right)$ such that $p^{*} \leq j(p)$ for all $p \in G^{i^{*}}$. Conditions in $\mathbb{P}_{i^{*}, i+2}$ can be seen as functions with domain $\left[i^{*}, i+2\right)$, so conditions in $j\left(\mathbb{P}_{i^{*}, i+2}\right)$ can be thought of as functions with domain $\left[i^{*}, j(i+2)\right)$. We recursively define $p^{*}(\alpha)$ for $\alpha \in$ $\left[i^{*}, j(i+2)\right)$. Suppose $\alpha$ is a successor ordinal and we have defined $p^{*} \uparrow\left[i^{*}, \alpha\right)$ such that $p^{*}\left\lceil\left[i^{*}, \alpha\right) \leq j(p) \uparrow\left[i^{*}, \alpha\right)\right.$ for all $p \in G$. The forcing at coordinate $\alpha$ in $j\left(\mathbb{P}_{i^{*}, i+2}\right)$ is a Levy collapse that is $j\left(\kappa_{i^{*}}\right)$-directed closed, and, in $M[G * H]$, $\left|G^{i^{*}}\right|=\kappa_{k^{*}}$, so there is $\dot{q}$ such that $p^{*} \uparrow\left[i^{*}, \alpha\right) \Vdash " \dot{q} \leq j(p)(\alpha)$ " for every $p \in G^{i^{*}}$. Let $p^{*}\left\lceil\alpha+1=p^{*}\lceil\alpha\ulcorner\dot{q}\right.$.

Now suppose $\alpha$ is a limit ordinal. We would like to thank Menachem Magidor and Yair Chayut for conveying the following argument. By Lemma 3.2, $\Vdash_{j\left(\mathbb{P}_{i^{*}}\right)}$ $" \sup \left(\left\{j(p)(\alpha)_{0} \mid p \in G^{i^{*}}\right\}\right)=\sup \left(\left\{j(g)(\alpha) \mid g \in \prod_{\ell \leq i} \kappa_{\ell+1} \cap V\right\}\right) "$. Let this common supremum be denoted by $\gamma$, and note that $\operatorname{cf}^{V}(\gamma) \leq \kappa_{i+1}$. We would like to let $p^{*}(\alpha)_{0}$ be forced by $p^{*}\left\lceil\alpha\right.$ to be equal to $\bigcup_{p \in G^{i^{*}}} j(p)(\alpha)_{0} \cup\{\gamma\}$. Let $A=\left\langle\vec{a}_{\ell}\right| \ell \in$ $\left.X_{i^{*}, i+2}\right\rangle$ be a sequence of names such that, for every $\ell \in X_{i^{*}, i+2}, \Vdash_{\mathbb{P}_{\ell}}$ " $\vec{a}_{\ell}$ is the 
enumeration of bounded subsets of $\kappa_{\ell+1}$ to be used in the forcing to shoot a club through the set of approachable points of $\kappa_{\ell+1}$." We must show that $\gamma$ is forced to be approachable with respect to $j(A)(\alpha)$.

Let $I_{\alpha}$ be $j\left(\mathbb{P}_{i^{*}, i+2}\right)_{i^{*}, \alpha}$-generic over $V[G * H]$ with $p^{*} \uparrow \alpha \in I_{\alpha}$. Note that the following facts hold.

(1) Since $\mathbb{P}_{k^{*}}$ satisfies the $\kappa_{k^{*}}^{+}$-c.c., if $g \in \prod_{\ell \leq i} \kappa_{\ell+1} \cap V\left[G_{k^{*}}\right]$, then there is $h \in$ $\prod_{\ell \leq i} \kappa_{\ell+1} \cap V$ such that, for all $i^{*} \leq \ell \leq i, g(\ell)<h(\ell)$.

(2) $\bar{V}\left[G * H * I_{\alpha}\right]$ is an extension of $V\left[G_{k^{*}}\right]$ by $\kappa_{k^{*}}$-distributive forcing.

(3) $M[G * H]$ is an extension of $M\left[G_{k^{*}}\right]$ by $\operatorname{Coll}\left(\kappa_{k^{*}},<j\left(\kappa_{i^{*}}\right)\right.$. Thus, any ordinal in the interval $\left[\kappa_{k^{*}}, \kappa_{i+2}\right]$ which is a regular cardinal in $V$ is an ordinal of cardinality and cofinality $\kappa_{k^{*}}$ in $M[G * H]$. Since $I_{\alpha}$ is generic for forcing that is $j\left(\kappa_{i^{*}}\right)$-distributive in $M[G * H]$, this remains true in $M\left[G * H * I_{\alpha}\right]$.

We can therefore, in $V\left[G * H * I_{\alpha}\right]$, find a sequence $\left\langle g_{\delta} \mid \delta<\kappa_{k^{*}}\right\rangle$ of elements of $\prod_{\ell \leq i} \kappa_{\ell+1} \cap V$ such that:

- For all $\eta<\delta<\kappa_{k^{*}}$ and $i^{*}<\ell \leq i, g_{\eta}(\ell)<g_{\delta}(\ell)$.

- For every $g \in \prod_{\ell \leq i} \kappa_{\ell+1}$, there is $\delta<\kappa_{k^{*}}$ such that, for every $i^{*}<\ell \leq i$, $g(\ell)<g_{\delta}(\ell)$

Notice that, due to the fact (2) listed above, $\left\langle g_{\delta} \mid \delta<\eta\right\rangle \in V\left[G_{k^{*}}\right]$ for every $\eta<\kappa_{k^{*}}$. Also, because of our technical assumption on the names $\vec{a}_{\ell}$ stated at the beginning of this proof, for every $\eta<\kappa_{k^{*}}$ and every $\ell \in X_{i^{*}, i+2}$, the index at which $\left\{g_{\delta}(\ell) \mid \delta<\eta\right\}$ is enumerated in $\vec{a}_{\ell}$ can be computed in $V\left[G_{k^{*}}\right]$. Thus, fact (1) listed above, there is $h_{\eta} \in \prod_{\ell \in X_{i^{*}, i+2}} \kappa_{\ell+1} \cap V$ such that, for every $\ell \in X_{i^{*}, i+2}$, the set $\left\{g_{\delta}(\ell) \mid \delta<\eta\right\}$ is enumerated in $\vec{a}_{\ell}$ with an index smaller than $h_{\eta}(\ell)$. It follows that the set $\left\{j\left(g_{\delta}\right)(\alpha) \mid \delta<\eta\right\}$ is enumerated in $j(A)(\alpha)$ with an index smaller than $j\left(h_{\eta}\right)(\alpha)<\gamma$.

By the closure properties of $M,\left\langle j\left(g_{\delta}\right)(\alpha) \mid \delta<\kappa_{k^{*}}\right\rangle \in M[G * H]$, and, by the previous paragraph, this sequence is easily seen to witness the approachability of $\gamma$ with respect to $j(A)(\alpha)$, so our definition of $p^{*}(\alpha)_{0}$ is valid. Finally, we define $p^{*}(\alpha)_{1}$ by noting that the second component of coordinate $\alpha$ in $j\left(\mathbb{P}_{i^{*}, i+2}\right)$ is $j\left(\kappa_{\omega+1}\right)>\kappa_{i+2}$-directed closed, so we can define $p^{*}(\alpha)_{1}$ to be a name for a lower bound for $\left\{j(p)(\alpha)_{1} \mid p \in G^{i^{*}}\right\}$.

We now have successfully completed the construction of $p^{*}$. Let $I$ be $j\left(\mathbb{P}_{i^{*}, i+2}\right)$ generic over $V[G * H]$ such that $p^{*} \in I$, and extend $j$ to $j: V[G] \rightarrow M[G * H * I]$.

Now suppose, for sake of contradiction, that $T$ does not reflect in $V[G]$. Then, in $M[G * H * I], j(T)$ is a stationary subset of $S_{\kappa_{k}}^{j\left(\kappa_{i+1}\right)}$ that does not reflect. In particular, letting $\eta=\sup \left(j " \kappa_{i+1}\right), j(T)$ does not reflect at $\eta$. Let $D$ be a club in $\eta$ disjoint from $j(T)$. Since $j$ " $\kappa_{i+1}$ is $<\kappa_{i^{*}}$-closed and unbounded in $\eta, D \cap j " \kappa_{i+1}$ is $<\kappa_{i^{*}}$-closed and unbounded in $\eta$ and is disjoint from $j(T)$. Thus, $E=j^{-1}$ " $(D \cap$ $j$ " $\left.\kappa_{i+1}\right)$ is a $<\kappa_{i^{*}}$-closed, unbounded subset of $\kappa_{i+1}$ that is disjoint from $T . E \in$ $V[G * H * I]$. However, as $I$ is generic for $j\left(\kappa_{i^{*}}\right)$-strategically closed forcing and $j\left(\kappa_{i^{*}}\right)>\kappa_{i+1}$, it must be the case that $E \in V[G * H]$. Thus, since $\kappa_{k}<\kappa_{i^{*}}$ and $T \subseteq S_{\kappa_{k}}^{\kappa_{i+1}}, E$ witnesses that $T$ is not stationary in $V[G * H]$. However, since $T$ is 
stationary in $V[G], A P_{\kappa_{i+1}}$ holds in $V[G]$, and $H$ is generic for $\kappa_{k^{*}}$-closed forcing, Fact 2.7 implies that $T$ remains stationary in $V[G * H]$. This is a contradiction, so $T$ does reflect in $V[G]$.

Case 2: $k>\omega$. Let $k^{*}$ be a successor ordinal such that $k<k^{*}<i$ and $i<\kappa_{k^{*}}$ (note that we can do this because $i<\kappa_{i}$ ), and let $i^{*}=k^{*}+1$. Let $X$ be the set of limit ordinals $\ell \in\left(i^{*}, i\right]$ such that $\ell<\kappa_{\ell}$. In $V[G]$, for $\ell \in X$, let $S_{\ell}$ be the stationary subset of $S_{\omega}^{\kappa_{\ell+1}}$ added by $\mathbb{S}_{\kappa_{\ell+1}}$. We would like to repeat the argument

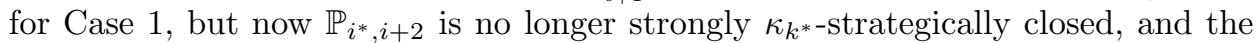
stationary sets $S_{\ell}$ present a concrete obstacle to lifting the relevant elementary embedding. Thus, we force to destroy the stationarity of the sets $S_{\ell}$ for $\ell \in X$. To this end, let $\mathbb{T}$ be the forcing poset whose conditions are functions $t$ such that $\operatorname{dom}(t)=X$ and, for all $\ell \in X, t(\ell)$ is a closed, bounded subset of $\kappa_{\ell+1}$ such that $t(\ell) \cap S_{\ell}=\emptyset$. If $s, t \in \mathbb{T}$, then $s \leq t$ if and only if, for every $\ell \in X, s(\ell)$ end-extends $t(\ell)$. Let $K$ be $\mathbb{T}$-generic over $V[G]$. The main reason for introducing $\mathbb{T}$ is that it allows us to construct long decreasing sequences of conditions from $\mathbb{P}_{i^{*}, i+2}$ that have lower bounds. This is made more precise in the following claim.

Claim 3.3. In $V\left[G_{i^{*}}\right]$, there is a dense subset of $\mathbb{P}_{i^{*}, i+2} * \mathbb{T}$ that is strongly $\kappa_{k^{*-}}$ strategically closed.

Proof. Let $\mathbb{U}$ be the set of $(p, \dot{t}) \in \mathbb{P}_{i^{*}, i+2} * \mathbb{T}$ such that there is $g \in \prod_{\ell \in X} \kappa_{\ell+1}$ such that, for all $\ell \in X, p\left\lceil\ell^{\frown} p(\ell)_{0} \Vdash_{\mathbb{P}_{i^{*}, \ell^{*}} \mathbb{Q}_{\vec{a}}}\right.$ " $\gamma^{p(\ell)_{1}}=g(\ell)+1$ " and $p \Vdash_{\mathbb{P}_{i^{*}, i+2}}$ $" \max (\dot{t}(\ell))=g(\ell) "$.

We first show that $\mathbb{U}$ is dense in $\mathbb{P}_{i^{*}, i+2} * \mathbb{T}$. Given $\left(p_{0}, \dot{t}_{0}\right) \in \mathbb{P}_{i^{*}, i+2} * \mathbb{T}$, find $p_{1} \leq p_{0}$ such that there is $h_{0} \in \prod_{\ell \leq i} \kappa_{\ell+1}$ such that, for every $\ell \in X, p_{1} \Vdash_{\mathbb{P}_{i^{*}, i+2}}$ " $\max \left(\dot{t}_{0}(\ell)\right)<h_{0}(\ell)$ ". Then, find $p_{2} \leq p_{1}$ such that, as in Lemma 3.2, $p_{2} \in D_{i^{*}, i+2}$, as witnessed by $h_{1} \in \prod_{\ell \leq i} \kappa_{\ell+1}$, where $h_{1}>h_{0}$. Now let $(p, \dot{t}) \leq\left(p_{2}, \dot{t}_{0}\right)$ be such that, for all $\ell \in X, p\lceil\ell\urcorner p(\ell)_{0} \Vdash_{\mathbb{P}_{i^{*}, \ell} * \mathbb{Q}_{\vec{a}}}$ " $\gamma^{p(\ell)_{1}}=h_{1}(\ell)+1$ " and $p \Vdash_{\mathbb{P}_{i^{*}, i+2}} " \max (\dot{t}(\ell))=$ $h(\ell)$ ". Then $(p, \dot{t}) \in \mathbb{U}$ and $(p, \dot{t}) \leq\left(p_{0}, \dot{t}_{0}\right)$, so $\mathbb{U}$ is dense in $\mathbb{P}_{i^{*}, i+1} * \mathbb{T}$.

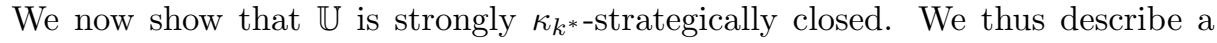
winning strategy for Player II in the game $G_{\kappa_{k^{*}}}^{*}(\mathbb{U})$. Suppose that $\beta<\kappa_{k^{*}}$ is an even successor ordinal and $\left\langle\left(p_{\alpha}, \dot{t}_{\alpha}\right) \mid \alpha<\beta\right\rangle$ has been played. All of the forcing iterands in $\mathbb{P}_{i^{*}, i+2}$ are already known to be strongly $\kappa_{k^{*}}$-strategically closed except for those of the form $\mathbb{S}_{\kappa_{\ell+1}}$ so, on all of the other coordinates, Player II plays at stage $\beta$ according to her winning strategy. To finish, at all $\ell \in X$, she simply lets $p_{\beta}(\ell)_{1}=p_{\beta-1}(\ell)_{1}$ and lets $\dot{t}_{\beta}=\dot{t}_{\beta-1}$. This is easily seen to describe a condition in $\mathbb{U}$ extending $\left(p_{\beta-1}, \dot{t}_{\beta-1}\right)$. If $\beta<\kappa_{k^{*}}$ is a limit ordinal and $\left\langle\left(p_{\alpha}, \dot{t}_{\alpha}\right) \mid \alpha<\beta\right\rangle$ has been played, we need to exhibit a greatest lower bound, $\left(p_{\beta}, \dot{t}_{\beta}\right)$, for this sequence of conditions. We define $p_{\beta}(\ell)$ recursively for $\ell \in\left[i^{*}, i+2\right)$. Let $p_{\beta}(\ell)$ (or $p_{\beta}(\ell)_{0}$, if $\ell \in X)$ be such that $p_{\beta} \uparrow \ell$ forces that $p_{\beta}(\ell)$ is the greatest lower bound of $\left\langle p_{\alpha}(\ell) \mid \alpha<\beta\right\rangle$. Such a greatest lower bound exists because Player II has been playing according to her winning strategy on coordinate $\ell$. For $\ell \in X$, we have two cases for $p_{\beta}(\ell)_{1}$. If $\left\langle\gamma^{p_{\alpha}(\ell)_{1}} \mid \alpha<\beta\right\rangle$ is eventually equal to some ordinal $\eta+1$, then let $p_{\beta}(\ell)_{1}$ be forced by $p_{\beta} \mid \ell^{\frown} p_{\beta}(\ell)_{0}$ to be equal to $\left(\bigcup_{\alpha<\beta} s^{p_{\alpha}(\ell)_{1}}, \eta+1\right)$. If $\left\langle\gamma^{p_{\alpha}(\ell)_{1}} \mid \alpha<\beta\right\rangle$ is not eventually constant, let $\eta=\sup \left(\left\{\gamma^{p_{\alpha}(\ell)_{1}} \mid \alpha<\beta\right\}\right)$ and again let $p_{\beta}(\ell)_{1}$ be forced by $p_{\beta} \mid \ell^{\frown} p_{\beta}(\ell)_{0}$ to be equal to $\left(\bigcup_{\alpha<\beta} s^{p_{\alpha}(\ell)_{1}}, \eta+1\right)$. The 
key point here is that, for each $\ell \in X$, we have ensured that $\left\{\max \left(\dot{t}_{\beta}(\ell)\right) \mid \alpha<\beta\right\}$ is a club in $\eta$ and witnesses that $\bigcup_{\alpha<\beta} s^{p_{\alpha}(\ell)_{1}}$ is not stationary in $\eta$. Finally, let $\dot{t}_{\beta}$ be such that, for all $\ell \in X$, letting $\eta$ be as in the definition of $p_{\beta}(\ell)_{1}$, $p_{\beta} \Vdash_{\mathbb{P}_{i *, i+2}}$ " $\dot{t}_{\beta}(\ell)=\bigcup_{\alpha<\beta} \dot{t}_{\alpha}(\ell) \cup\{\eta\}$ ". It is easily seen that $\left(p_{\beta}, \dot{t}_{\beta}\right) \in \mathbb{U}$ and is a greatest lower bound of $\left\langle\left(p_{\alpha}, \dot{t}_{\alpha}\right) \mid \alpha<\beta\right\rangle$.

As in Case $1, \kappa_{i^{*}}$ is still supercompact in $V\left[G_{k^{*}}\right]$, so let $j: V\left[G_{k^{*}}\right] \rightarrow M\left[G_{k^{*}}\right]$ witness that $\kappa_{i^{*}}$ is $\kappa_{i+2^{-}}$-supercompact. Since $\mathbb{P}_{i^{*}, i+2} * \mathbb{T}$ has a dense, strongly $\kappa_{k^{*}-}$ strategically closed subset, we can use Fact 2.2 to lift $j$ to $j: V\left[G_{i^{*}}\right] \rightarrow M[G * K * H]$, where $H$ is generic over $V[G * K]$ for $\kappa_{k^{*}}$-closed forcing. We now define a master condition $p^{*} \in j\left(\mathbb{P}_{i^{*}, i+2}\right)$ such that $p^{*} \leq j(p)$ for every $p \in G^{i^{*}}$. This is done exactly as in Case 1 except for the following: if $\ell \in X$, let $\eta_{\ell}=j^{*} \kappa_{\ell+1}$. Then $p^{*}(\ell)_{1}$ is defined so that it is forced by $p^{*}\left\lceil\ell^{\frown} p^{*}(\ell)_{0}\right.$ to be equal to $\left(\bigcup_{p \in G^{i^{*}}} s^{p(\ell)_{1}}, \eta+1\right)$. Note that it is forced that $\sup \left(s^{p^{*}(\ell)_{1}}\right)=\eta_{\ell}$ and $p^{*}(\ell)_{1} \in j\left(\mathbb{S}_{\kappa_{\ell+1}}\right)$, since $\bigcup_{t \in K} j(t)(\ell)$ is forced to be a club in $\eta$ disjoint from $s^{p^{*}(\ell)_{1}}$. Also, since $i<\operatorname{crit}(j)$, elements of $j\left(\mathbb{P}_{i^{*}, i+2}\right)$ can also be thought of as functions with domain $\left[i^{*}, i+2\right)$, so this finishes the definition of $p^{*}$.

Thus, letting $I$ be $j\left(\mathbb{P}_{i^{*}, i+2}\right)$-generic over $V[G * K * H]$ with $p^{*} \in I$, we can lift our embedding to $j: V[G] \rightarrow M[G * K * H * I]$. If $T$ does not reflect in $V[G]$, then, as before, we can find a club $E$ in $\kappa_{i+1}$ such that $E \in V[G * K]$ and $E \cap T=\emptyset$. Thus, we will reach a contradiction and finish the proof if we demonstrate the following claim.

Claim 3.4. $T$ remains stationary in $V[G * K]$.

Proof. Work in $V[G]$. Let $t_{0} \in \mathbb{T}$, and let $\dot{D}$ be a $\mathbb{T}$-name for a club in $\kappa_{i+1}$. We will find $t \leq t_{0}$ such that $t \Vdash_{\mathbb{T}}$ " $\dot{D} \cap \check{T} \neq \emptyset$ ". Let $\theta$ be a sufficiently large regular cardinal. Since $A P_{\kappa_{i}}$ holds (it was forced by $\mathbb{P}_{i, i+1}$ ), we can find an internally approachable continuous chain of elementary substructures of $H(\theta),\left\langle M_{\xi} \mid \xi<\kappa_{k}\right\rangle$ such that:

- $\mathbb{T},\left\{\kappa_{\ell} \mid \ell \leq i+1\right\}, t_{0}, \dot{D} \in M_{0}$.

- $\kappa_{k}^{+} \subset M_{0}$.

- For all $\xi<\kappa_{k},\left|M_{\xi}\right|<\kappa_{k^{*}}$.

- Letting $M=\bigcup_{\xi<\kappa_{k}} M_{\xi}, \sup \left(M \cap \kappa_{i+1}\right) \in T$.

For all $\ell \in X$, let $\lambda_{\ell}=\sup \left(M \cap \kappa_{\ell+1}\right)$. For each $\ell \in X, \operatorname{cf}\left(\lambda_{\ell}\right)=\kappa_{k}$ and $\left\langle\sup \left(M_{\xi} \cap\right.\right.$ $\left.\kappa_{\ell+1}\right)\left|\xi<\kappa_{k}\right\rangle$ enumerates a club $C_{\ell}^{0}$ in $\lambda_{\ell}$, all of whose initial segments are in $M$. In fact, since $\kappa_{k}^{+} \subset M$ every bounded subset of $C_{\ell}^{0}$ is in $M$. Also, for all $\ell \in X$, since $k>\omega, S_{\ell}$ does not reflect at $\lambda_{\ell}$, so there is a club $C_{\ell}^{1}$ in $\lambda_{\ell}$ such that $C_{\ell}^{1} \cap S_{\ell}=\emptyset$. For all $\ell \in X$, let $C_{\ell}=C_{\ell}^{0} \cap C_{\ell}^{1}$. By the preceding, we have that $C_{\ell}$ is a club in $\kappa_{\ell+1}$ disjoint from $S_{\ell}$, all of whose initial segments are in $M$. In fact, again since $\kappa_{k}^{+} \subset M$, any sequence $\left\langle C_{\ell}^{\prime} \mid \ell \in X\right\rangle$ such that each $C_{\ell}^{\prime}$ is an initial segment of $C_{\ell}$ is in $M$. For each $\ell \in X$, let $\left\langle\eta_{\alpha}^{\ell} \mid \alpha<\kappa_{k}\right\rangle$ be an increasing enumeration of $C_{\ell}$.

We now construct a descending sequence $\left\langle t_{\alpha} \mid \alpha<\kappa_{k}\right\rangle$ of conditions in $\mathbb{T} \cap M$. In fact, any initial segment of the construction can be computed inside $M$, so any initial segment of the sequence of conditions will also be in $M$.

$t_{0}$ has already been given. Suppose $t_{\alpha} \in M$ is given. We will construct $t_{\alpha+1}$. First, let $t_{\alpha}^{\prime}$ be the $<_{\theta}$-least condition in $\mathbb{T}$ such that $t_{\alpha}^{\prime} \leq t_{\alpha}$ and there is $\gamma>\eta_{\alpha}^{i}$

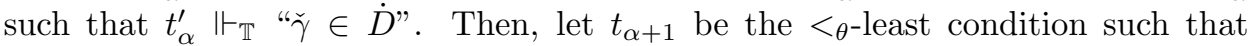


$t_{\alpha+1} \leq t_{\alpha}^{\prime}$ and, for every $\ell \in X$, there is $\beta>\alpha$ such that $\max \left(t_{\alpha+1}(\ell)\right)=\eta_{\beta}^{\ell}$. Note that the calculation of $t_{\alpha+1}$ only requires sufficiently long initial segments of the clubs $C_{\ell}$, so $t_{\alpha+1} \in M$.

If $\beta<\kappa_{k}$ is a limit ordinal and $\left\langle t_{\alpha} \mid \alpha<\beta\right\rangle$ has been constructed, then, for each $\ell \in X$, let $\delta_{\ell}=\sup \left(\bigcup_{\alpha<\beta} t_{\alpha}(\ell)\right)$ and define $t_{\beta}$ by letting $t_{\beta}(\ell)=\bigcup_{\alpha<\beta} t_{\alpha}(\ell) \cup\left\{\delta_{\ell}\right\}$. $t_{\beta}$ is a valid condition in $\mathbb{T}$ since, for each $\ell \in X, \delta_{\ell}$ is in $C_{\ell}$ and hence not in $S_{\ell}$. Also, the calculation of $t_{\beta}$ only requires $\left\langle t_{\alpha} \mid \alpha<\beta\right\rangle$, calculation of which itself only requires sufficiently long initial segments of the $C_{\ell}$ 's, so $t_{\beta} \in M$.

Finally, define $t \in \mathbb{T}$ by, for each $\ell \in X$, letting $t(\ell)=\bigcup_{\alpha<\kappa_{k}} t_{\alpha}(\ell) \cup\left\{\lambda_{\ell}\right\}$. Each $\lambda_{\ell}$ has cofinality $\kappa_{k}>\omega$, so $\lambda_{\ell} \notin S_{\ell}$ and thus $t$ is in fact in $\mathbb{T}$. Also, $t \leq t_{\alpha}$ for all $\alpha<\kappa_{k}$, so $t \Vdash_{\mathbb{T}}$ " $\check{\lambda}_{i} \in \dot{D}$ ", so, in particular, $t \Vdash_{\mathbb{T}}$ " $\dot{D} \cap \check{T} \neq \emptyset$ ".

It remains open whether we can find a model in which bounded stationary reflection holds at the successor of every singular cardinal greater $\aleph_{\omega}$. The difficulty in dealing with successors of cardinal fixed points lies in the fact that, if we are unable to use an elementary embedding with critical point in the interval $\left(i, \kappa_{i}\right)$, then our proof of Claim 3.4 does not work. By suitably varying the cofinalities of the points at which the stationary sets $S_{\ell}$ are allowed to reflect, we can obtain a model in which bounded stationary reflection holds at the successors of all singular cardinals greater than $\aleph_{\omega}$ which are not limits of cardinal fixed points, but it seems that this approach cannot be extended to attain a truly global result.

\section{REFERENCES}

[1] Y. Chayut. Stationary reflection and the approachability property. Master's thesis, Hebrew University of Jerusalem, 2013.

[2] T. Eisworth. Private communication.

[3] T. Eisworth. Successors of singular cardinals. In Handbook of set theory, pages 1229-1350. Springer, 2010.

[4] T. Eisworth. Simultaneous reflection and impossible ideals. Journal of Symbolic Logic, $77(4): 1325-1338,2012$.

[5] T. Jech. Set theory, the third millennium, revised and expanded ed. Springer Monographs in Mathematics, Springer-Verlag, Berlin, 2003.

[6] R. B. Jensen. The fine structure of the constructible hierarchy. Annals of Mathematical Logic, $4(3): 229-308,1972$.

[7] M. Magidor. Reflecting stationary sets. Journal of Symbolic Logic, 47(4):755-771, 1982.

[8] S. Shelah. Reflecting stationary sets and successors of singular cardinals. Archive for Mathematical Logic, 31(1):25-53, 1991.

[9] R. Solovay, W. Reinhardt, and A. Kanamori. Strong axioms of infinity and elementary embeddings. Annals of Mathematical Logic, 13(1):73-116, 1978.

Department of Mathematical Sciences, Carnegie Mellon University, Pittsburgh, PA 15213

E-mail address: jcumming@andrew.cmu.edu

Einstein Institute of Mathematics, Hebrew University of Jerusalem, Jerusalem, 91904, ISRAEL

E-mail address: clambiehanson@math.huji.ac.il 